\title{
The utility of p16 immunostaining in cutaneous keratinocytic lesion
}

\author{
Ran Hong \\ Department of Pathology, College of Medicine, Chosun University, Gwangju, Korea
}

Received January 23, 2019

Revised February 13, 2019

Accepted February 13, 2019

Corresponding author

Ran Hong

Department of Pathology, College

of Medicine, Chosun University,

365, Philmundae-ro, Dong-gu,

Gwangju 61453, Korea

Tel: +82-62-230-6356

Fax: +82-62-226-5860

E-mail: nanih@chosun.ac.kr

ORCID:

https://orcid.org/0000-0001-7011-5215

\begin{abstract}
Several kinds of atypical keratinocytic lesions including seborrheic keratosis (SK), actinic keratosis (AK), Bowen's disease (BD) and squamous cell carcinoma ( $\mathrm{SqCC}$ ) are common lesion in pathologic practice. While most of them show characteristic histological feature, occasionally we meet the diagnostic difficulty by overlapping or mixed histologic features. The aim of this study was to evaluate the immunostaining pattern of p16 (INK4a) in SK, AK, BD and SqCC, and identify the utility of p16 immunohistochemical staining in differential diagnosis of above cutaneous keratinocytic lesions. We studied 50 cases of keratinocytic lesions (20 examples of SK and 10 cases of each of $\mathrm{AK}, \mathrm{BD}$ and $\mathrm{SqCC})$. The cases were stained for $\mathrm{p} 16$. Ninety percent of $\mathrm{BD}$ cases showed strong block-positivity and typical SK not show reactivity with p16, just only one case showed patch staining pattern. In AK, only one case showed block-positivity and another cases showed patchy staining pattern. In SqCC, only 3 out of 10 cases showed strong staining pattern. This study demonstrated that the expression of p16 is strongly associated with the progression of $\mathrm{BD}$ $(p<0.001)$. This staining pattern was not seen in AK and SK. Accordingly, immunohistochemistry for p16 is a useful test in the differential diagnosis of intra-epidermal atypical keratinocytic lesion. However, despite the statistical significance $(p<0.001)$, our small series suggests that as not all typical cases of $\mathrm{BD}$ are positive with $\mathrm{p} 16$, negative $\mathrm{p} 16$ staining alone cannot be used to classify atypical keratinocytic lesions, and the finding of hematoxylin and eosin staining remains paramount.
\end{abstract}

Keywords: p16; Immunohistochemistry; Skin; Keratinocyte; Bowen disease

\section{INTRODUCTION}

The cutaneous keratinocytic lesion such as seborrheic keratosis (SK), actinic keratosis (AK), Bowen's disease (BD) and Squamous cell carcinoma (SqCC) are common lesion in pathologic practice. While $\mathrm{BD}$ and $\mathrm{AK}$ are premalignant lesion to SqCC, SK is benign, so differentiation of these lesions is clinically important, however, the distinction between them is sometimes diagnostically challenging. For example, separation of AK from BD in small eroded punch biopsied specimens, lesions showing architectural features of SK with atypical cytology, either as a reactive change secondary to irritation, or true dysplastic change in a SK, lesions of $\mathrm{BD}$ with subtle cytological atypia, which can mimic BD [1-4]. $\mathrm{p} 16^{\mathrm{INK} 4 \mathrm{a}}(\mathrm{p} 16)$ is a tumor suppressor gene, plays an important role in cell cycle progression and cellular differentiation [4]. As a cyclin-dependent kinase inhibitor, p16 ${ }^{\mathrm{INK} 4 a}$ encoded by the INK4a/ARF gene located on chromosome 9p21 is part of the retinoblastoma protein (pRB)-mediated control of the G1-S-phase transition and triggers cell cycle arrest in the course of the cellular differentiation process, specifically blocks CDK4 and CDK6 and decreased phos- 
phorylation of $\mathrm{pRB}$ with subsequent arrest in the $\mathrm{G} 1$ phase of the cell cycle. Normally, terminally differentiated cells, $\mathrm{p} 16^{\mathrm{INK} 4 \mathrm{a}}$ is expressed at low levels, typically not detected by immunohistochemistry (IHC) [4,5]. However, sometimes, overexpression of $\mathrm{p} 16^{\mathrm{INK} 4 \mathrm{a}}$ protein has been demonstrated to contribute to cell cycle deregulation and cellular transformation. Negri et al. [6] have demonstrated strong over-expression of $\mathrm{p} 16^{\mathrm{INK} 4 \mathrm{a}}$ in pre-cancerous lesion and cancer to be closely related at the molecular level to E7 oncoprotein expression from the human papillomavirus (HPV). Immunohistochemistry (IHC) for p16 is a well established tool in the diagnosis of high-grade dysplasia in the uterine cervix. Several studies for cutaneous lesion have reported that BD often immunoreactivity for p16, AK is variable, and SK is negative. These results suggest that $\mathrm{p} 16 \mathrm{IHC}$ may be valuable in the differential diagnosis of BD with AK and SK.

Herein, we compared the immunostaining results p16 in SK, AK, BD and SqCC to determine whether it could be valuable tool in diagnostically difficult cases in pathologic practice.

\section{MATERIALS AND METHODS}

\section{Samples}

In total, 50 samples of keratinocytic lesion composed of 20 cases of SK, 10 of AK, 10 of BD, and 10 of SqCC were obtained from punch biopsy performed at the Department of Dermatology, Chosun University Hospital (Gwangju, Korea) between February 2016 and December 2017.

\section{Histopathological analysis}

We re-evaluated the medical records and the tissue slide files at the Department of Pathology, College of Medicine, Chosun University (Gwangju, Korea). The diagnosis of keratinocytic lesion were re-confirmed. The examined tissues were fixed in $10 \%$ neutral formalin and the prepared paraffin-embedded tissues were sectioned (4-5 $\mu \mathrm{m}$ in thickness).

\section{1) Hematoxylin and eosin (H\&E) staining}

H\&E staining (Ventana Medical Systems, Tucson, AZ, USA) was performed according to the standard procedure and the sections were examined under a light microscope (Olympus BX51; Olympus Corporation, Tokyo, Japan). By review of the H\&E slides, the histological diagnosis was reevaluated.

\section{2) Immunohistochemical staining}

All specimens were tested using mouse monoclonal antip16 ${ }^{\text {INK4a }}$ (CINtec p16 Histology, Ventana). The staining process was performed according to the manufacturer's protocol of the NexES autoimmunostainer (Ventana Medical Systems). Briefly, the 4- $\mu \mathrm{m}$ sections obtained following formalin fixation and paraffin embedding were de-paraffinized in xylene and then rehydrated with distilled water through a graded series of ethanol solutions. The sections were then placed in a glass jar with $10 \mathrm{mmol} / \mathrm{L}$ citrate buffer ( $\mathrm{pH}$ 6.0) and were irradiated in a microwave oven for 15 $\min$ at $99^{\circ} \mathrm{C}$. The sections were allowed to cool in the jar at room temperature for $20 \mathrm{~min}$. The slides were then rinsed with Tris-buffered saline and, subsequent to quenching the endogenous peroxidase activity in $0.3 \%$ hydrogen peroxide for $10 \mathrm{~min}$. The slides were then washed as described, and immunohistochemistry was performed using the NexES autoimmunostainer. The resultant staining pattern for each

Table 1. Summary of clinical factors and immunostaining result of p16

\begin{tabular}{|c|c|c|}
\hline & Factor & $N(\%)$ \\
\hline \multirow[t]{5}{*}{ Age } & $50 s$ & $6(12.0)$ \\
\hline & $60 s$ & $9(18.0)$ \\
\hline & $70 \mathrm{~s}$ & $16(32.0)$ \\
\hline & $80 s$ & $16(32.0)$ \\
\hline & 90 s & $3(6.0)$ \\
\hline \multirow[t]{12}{*}{ Site } & Temple & 6 \\
\hline & Back & 2 \\
\hline & Buttock & 1 \\
\hline & Chest & 3 \\
\hline & Scalp & 6 \\
\hline & Auricle & 3 \\
\hline & Cheek & 10 \\
\hline & Abdomen & 3 \\
\hline & Nose & 3 \\
\hline & Perioral & 6 \\
\hline & Neck & 1 \\
\hline & Extremity & 4 \\
\hline \multirow[t]{4}{*}{ Diagnosis } & Seborrheic keratosis & $20(40.0)$ \\
\hline & Actinic keratosis & $10(20.0)$ \\
\hline & Bowen disease & $10(20.0)$ \\
\hline & Squamous cell carcinoma & $10(20.0)$ \\
\hline \multirow{4}{*}{$\begin{array}{l}\text { P16(+) } \\
\text { (block-positive) }\end{array}$} & Seborrheic keratosis & $0 / 10(0)$ \\
\hline & Actinic keratosis & $1 / 10(10.0)$ \\
\hline & Bowen disease & $9 / 10(90.0)$ \\
\hline & Squamous cell carcinoma & $3 / 10(30.0)$ \\
\hline
\end{tabular}


case was read as positive when the whole layer of the keratinocytic atypia is strongly stained (block-positive).

\section{Statistical analysis}

Statistical analysis was performed using SPSS 12.0 software (SPSS, Inc., Chicago, IL, USA). The $\chi^{2}$ test and Fisher's exact test were used to demonstrate the association between the p16 expression and the diagnosis. $\mathrm{P}<0.05$ was considered to be statistically significant.

\section{RESULTS}

The clinical factors and the results of immunohistochemistry for P16 are summarized in Table 1. Of the various cutaneous keratinocytic lesions, $90 \%$ of cases ( $9 / 10$ cases) of $\mathrm{BD}$ showed overexpression of p16 (Fig. 1). And there is distinct sparing of the palisading basal cell (PBC) layer in $\mathrm{BD}$ (Fig. 1B). However, typical SK was not reactive for p16, only one case showed patch staining pattern. Ina one case of AK, focal block-positivity was found, and another cases showed patchy staining pattern (Fig. 2). In 10 cases of SqCC, only 3 cases showed strong immunoreactivity (Fig. 3).

\section{DISCUSSION}

We identified a obvious difference in the p16 staining pattern between $\mathrm{BD}$ and $\mathrm{AK} / \mathrm{SK} / \mathrm{SqCC}$. In $\mathrm{BD}$, characteristically, they show strong block positivity of the epidermal
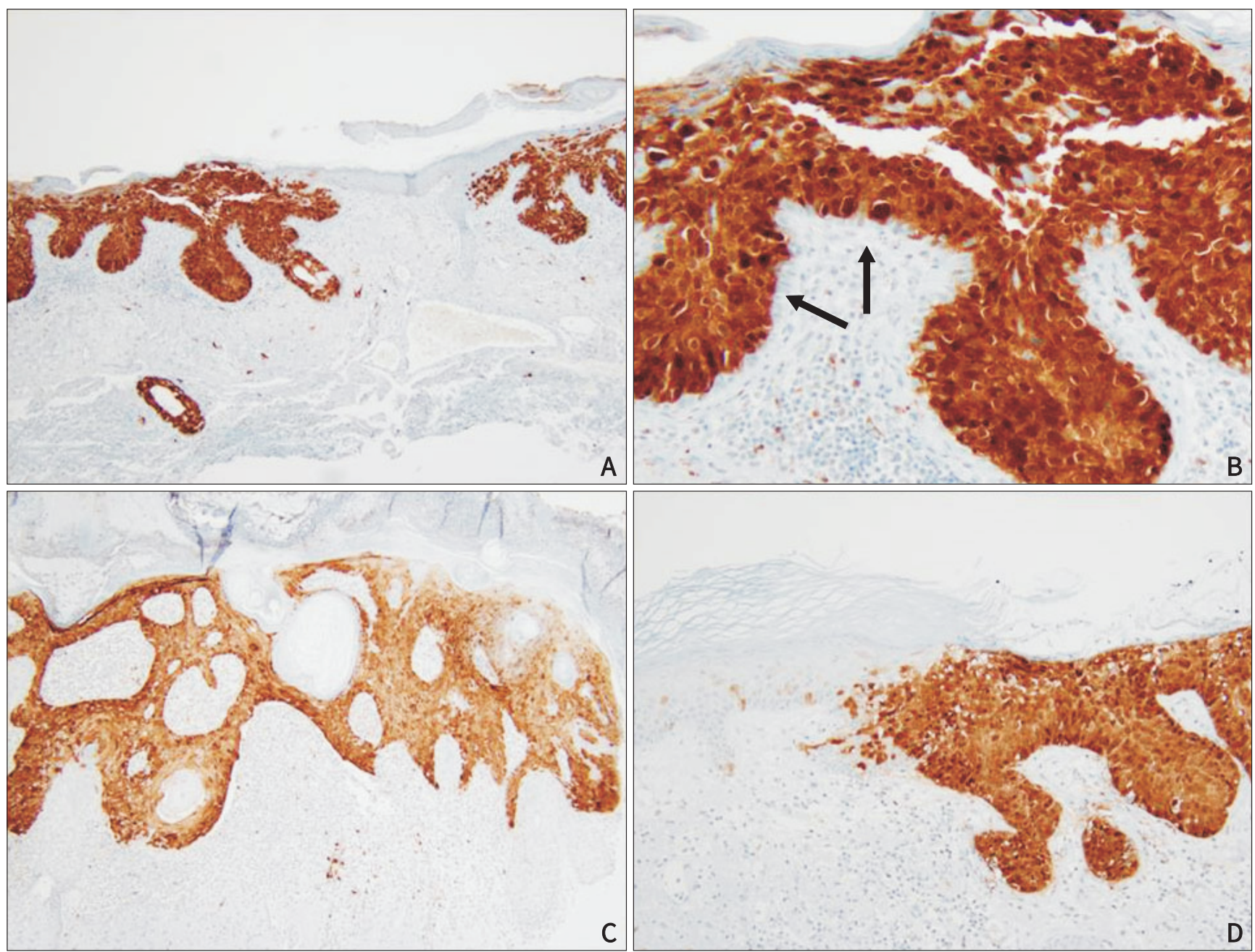

Fig. 1. Immunohistochemical staining of p16 in 2 cases of Bowen's disease (A \& B, C \& D). (A, B) In a first case, p16 staining shows strong blockpositivity of all lesion with distinct sparing of the palosaded basal cells (B, arrow) and central normal area. $(C, D)$ In another case of Bowen' disease, strond immunoreactivity is identified $(\mathrm{A}: \times 10, \mathrm{~B}: \times 40, \mathrm{C}, \mathrm{D}: \times 20)$. 


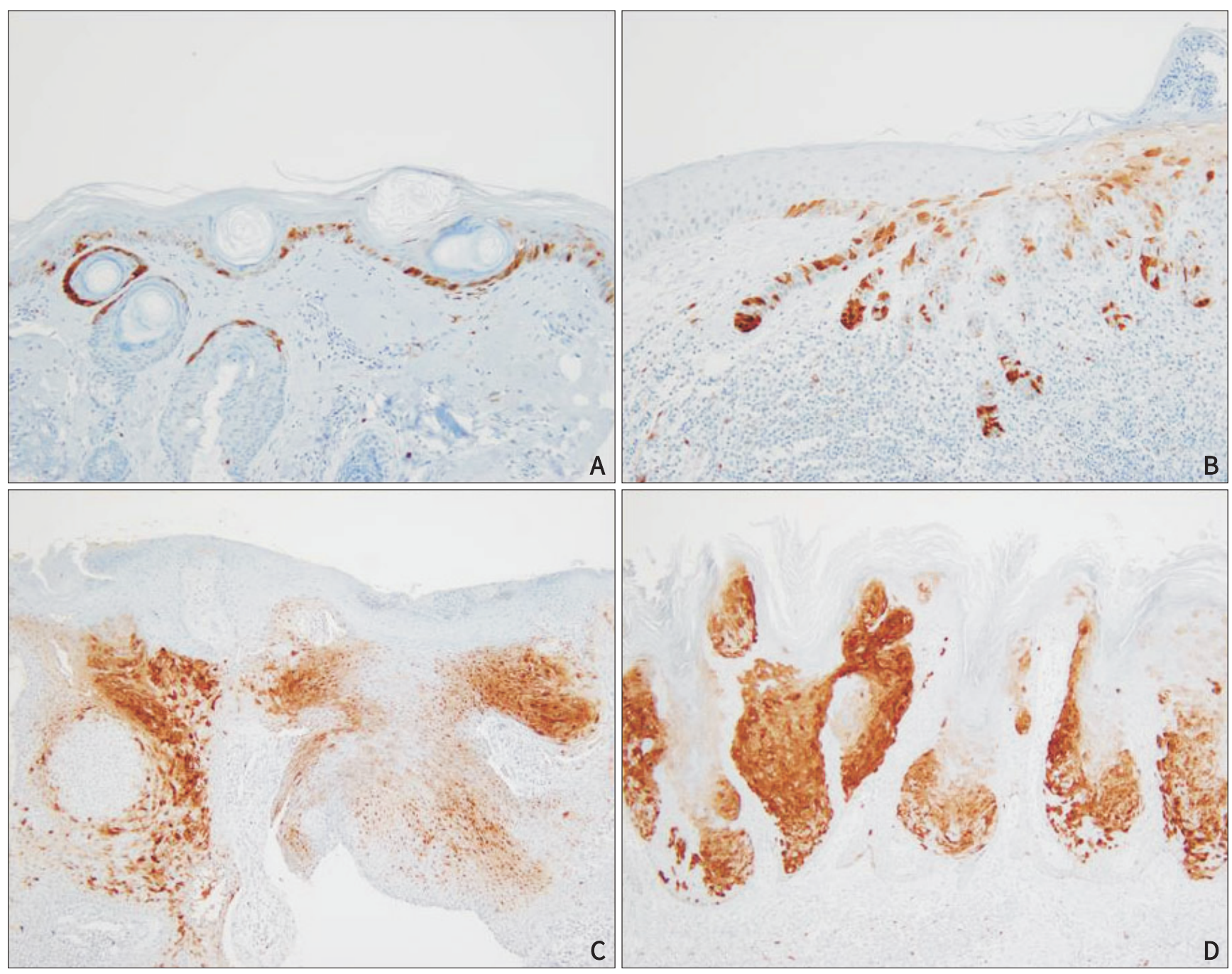

Fig. 2. Immunohistochemical staining of p16 in actinic keratosis. (A-C) Scattered single cells staining patterns are shown. (D) In a one case, block positivity is identified $(A: \times 10, B-D: \times 20)$.

lesion with basal layer sparing in some cases, that is, a layer of cytologically bland keratinocytes below the atypical neoplastic keratinocytes. Although it is not universal phenomenon, which may have diagnostic value in differentiating $\mathrm{BD}$.

Although the mechanism of $\mathrm{p} 16$ overexpression in $\mathrm{BD}$ remains obscure, recent studies demonstrated the effect of UV exposure [7]. Conscience et al. [7] showed p16 expression in $68 \%$ of SqCC on sun exposed sites compared to $23 \%$ of nonsun exposed sites, however, Blokx et al. [5] insisted that sun exposure has no correlation with p16 overexpression.

In dysplasia of uterine cervix, the function as a tumor suppressor of $\mathrm{pRB}$ is inactivated by the high riSK HPV E7 oncoprotein, which indirectly lead to overexpression of p16 $[8,9]$. However, there is no correlation between HPV DNA and p16 expression in the cutaneous lesion [10]. Nilsson et al. [11] suggested that other genetic aberration beside high riSK HPV E7 oncoprotein may lead inactivation of pRB, and which result in overexpression of $\mathrm{p} 16$. However, the precise nature of the underlying genetic aberration remains obscure.

Conclusively, we have identified consistent pattern of strong expressions in $\mathrm{BD}$ with a characteristic pattern of $\mathrm{PBC}$ sparing in some cases of $\mathrm{BD}$, which is not identified in $\mathrm{AK}$ and SK. p16 expression and identification of $\mathrm{PBC}$ sparing pattern can be useful for supporting a diagnosis of $\mathrm{BD}$ in pathologically difficult cases and separating BD from irritated or inflammatory AK or SK showing cytological atypia. 

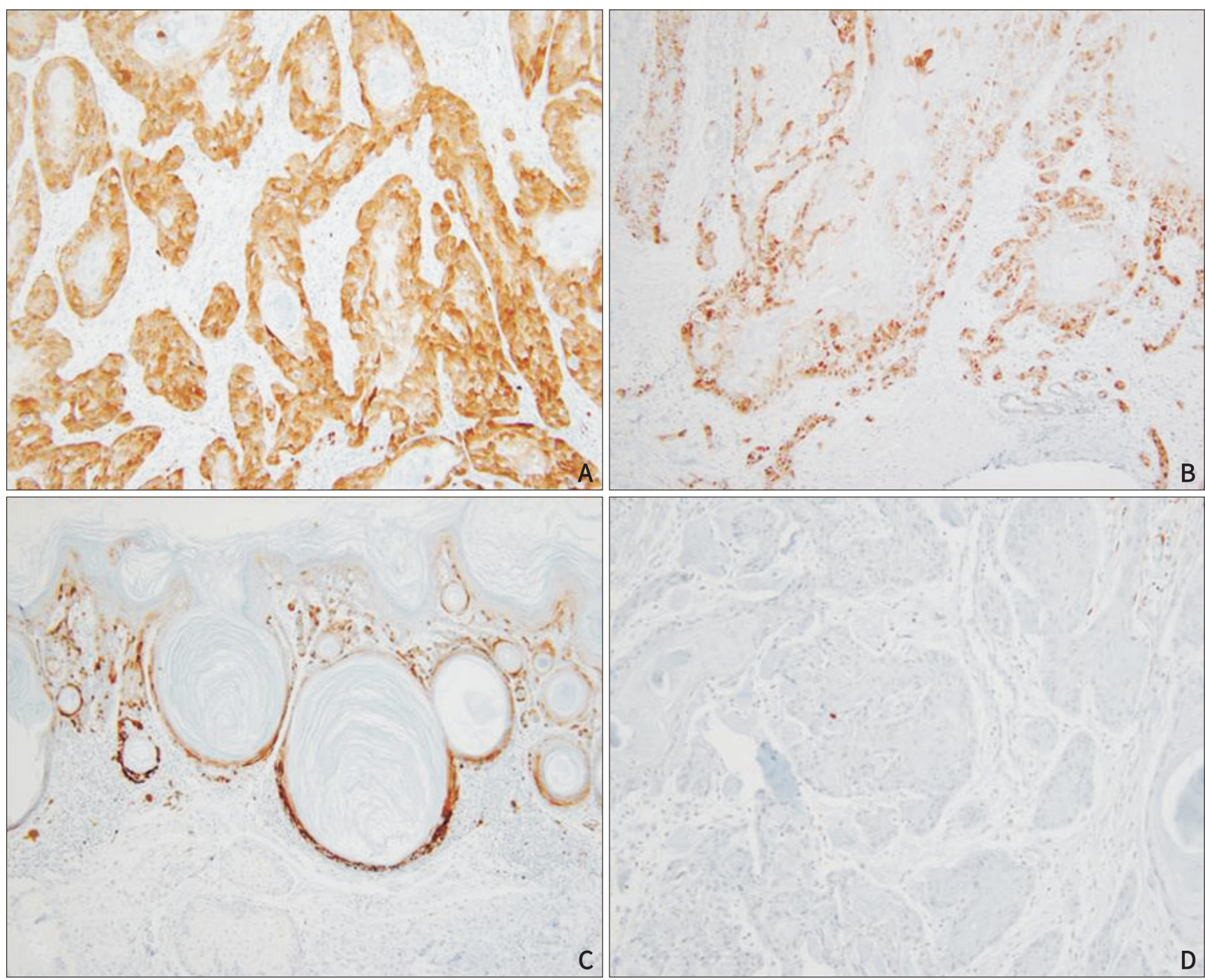

Fig. 3. Various immunohistochemical staining pattern of $p 16$ in squamous cell carcinoma. (A) Block-positive, (B, C) Patchy staining, (D) Negative (A, B, D: $\times$ 40, C: $\times 10)$.

\section{ACKNOWLEDGMENTS}

This study was supported by research funds from Chosun University Hospital, Republic of Korea, 2015.

\section{CONFLICT OF INTEREST}

No potential conflict of interest relevant to this article was reported.

\section{REFERENCES}

1. Bloch PH. Transformation of seborrheic keratosis into Bowen's disease. J Cutan Pathol 1978;5:361-7.
2. Monteagudo JC, Jorda E, Terencio C, Llombart-Bosch A. Squamous cell carcinoma in situ (Bowen's disease) arising in seborrheic keratosis: three lesions in two patients. J Cutan Pathol 1989;16:348-52.

3. Weedon D. Weedon's skin pathology. 3rd ed. Brisbane: Elsevier; 2010.

4. Harvey NT, Leecy T, Wood BA. Immunohistochemical staining for p16 is a useful adjunctive test in the diagnosis of Bowen's disease. Pathology 2013;45:402-7.

5. Blokx WA, de Jong EM, de Wilde PC, Bulten J, Link MM, Ruiter DJ, et al. P16 and p53 expression in (pre)malignant epidermal tumors of renal transplant recipients and immunocompetent individuals. Mod Pathol 2003;16:869-78.

6. Negri G, Vittadello F, Romano F, Kasal A, Rivasi F, Girlando S, et al. p16INK4a expression and progression risk of low-grade intraepithelial neoplasia of the cervix uteri. Virchows Arch 
2004;445:616-20.

7. Conscience I, Jovenin N, Coissard C, Lorenzato M, Durlach A, Grange F, et al. P16 is overexpressed in cutaneous carcinomas located on sun-exposed areas. Eur J Dermatol 2006;16:518-22.

8. Horn LC, Reichert A, Oster A, Arndal SF, Trunk MJ, Ridder R, et al. Immunostaining for p16INK4a used as a conjunctive tool improves interobserver agreement of the histologic diagnosis of cervical intraepithelial neoplasia. Am J Surg Pathol 2008;32:502-12.

9. Tam SW, Shay JW, Pagano M. Differential expression and cell cycle regulation of the cyclin-dependent kinase 4 inhibitor p16Ink4. Cancer Res 1994;54:5816-20.

10. Willman JH, Heinz D, Golitz LE, Shroyer KR. Correlation of p16 and pRb expression with HPV detection in Bowen's disease. J Cutan Pathol 2006;33:629-33.

11. Nilsson K, Svensson S, Landberg G. Retinoblastoma protein function and p16INK4a expression in actinic keratosis, squamous cell carcinoma in situ and invasive squamous cell carcinoma of the skin and links between p16INK4a expression and infiltrative behavior. Mod Pathol 2004;17:1464-74. 